\title{
A Derivative-Free Liu-Storey Method for Solving Large-Scale Nonlinear Systems of Equations
}

\author{
Zhenhua Su $\mathbb{D D}^{1}$ and $\operatorname{Min} \mathrm{Li}^{2}$ \\ ${ }^{1}$ College of Mathematics and Statistics, Hunan Normal University, Changsha 410081, China \\ ${ }^{2}$ Department of Mathematics and Applied Mathematics, Huaihua University, Huaihua 418000, China
}

Correspondence should be addressed to Zhenhua Su; szh820@163.com

Received 22 June 2020; Revised 6 October 2020; Accepted 16 October 2020; Published 29 October 2020

Academic Editor: Chaudry M. Khalique

Copyright ( $) 2020$ Zhenhua Su and Min Li. This is an open access article distributed under the Creative Commons Attribution License, which permits unrestricted use, distribution, and reproduction in any medium, provided the original work is properly cited.

In this paper, a descent Liu-Storey conjugate gradient method is extended to solve large-scale nonlinear systems of equations. Based on certain assumptions, the global convergence property is obtained with a nonmonotone line search. The proposed method is suitable to solve large-scale problems for the low-storage requirement. Numerical experiment results show that the new method is practically effective.

\section{Introduction}

Consider the following nonlinear system of equations:

$$
F(x)=0 \text {, }
$$

where $\left(F: R^{n} \longrightarrow R^{n}\right)$ has continuous partial derivatives. In the past few decades, Newton's methods have been widely used to solve problem (1) for their fast convergence speeds, see [1-10]. Newton's methods determine the search direction by solving a linear system of equations, in which the coefficient matrix is the Jacobian matrix of $F$ or an approximation of it. It is expensive to compute the coefficient matrix and solve the linear system of equations at each iteration. Therefore, Newton's methods are not suitable for solving a large-scale problem in which the Jacobian matrix of $F$ is unavailable or needs massive storage space. To overcome this shortcoming of Newton's methods, in this paper, we attempt to develop a numerical algorithm based on a descent Liu-Storey conjugate gradient method and a nonmonotone line research.

Recently, La Cruz and Raydan [11] designed the Spectral Algorithm for Nonlinear Equations (SANE) to solve (1) and analyzed the convergence property based on a modified Grippo-Lampariello-Lucidi (GLL) [12] nonmonotone line search. However, at each iteration, SANE needs to compute a directional derivative or an approximation of it. To improve the efficiency of the SANE algorithm, La Cruz et al. [13] proposed the derivative-free SANE (DF-SANE) algorithm by using a derivative-free nonmonotone line search method. Numerical experiment results show that the DF-SANE is efficient for solving large-scale problems. Similar to the DFSANE method, Cheng and Li [14] developed the N-DFSANE method for solving large-scale nonlinear systems of equations by combining the spectral residual method and the Zhang-Hager [15] nonmonotone line search. The global convergence of the method was established based on some mild conditions.

If the continuously differentiable mapping $F: R^{n} \longrightarrow R^{n}$ is the gradient of $f: R^{n} \longrightarrow R$, then (1) is the first-order necessary optimality condition of the optimization problem

$$
\min f(x), \quad x \in R^{n},
$$

namely, we can get the solution of equation (1) by solving optimization problem (2). Therefore, it is reasonable to extend the numerical algorithms for solving (2) to solve large-scale nonlinear systems of equations. In this paper, we develop a derivative-free method for solving (1) based on a descent nonlinear conjugate gradient method. 
Nonlinear conjugate gradient methods are efficient for solving large-scale unconstrained optimization problems for their low-memory requirements and excellent convergence properties. Among the nonlinear conjugate gradient methods, the Hestenes-Stiefel (HS), Polak-Ribière-Polyak (PRP), and Liu-Storey (LS) method [16-19] are the most famous methods for the excellent performance in practice. These methods have been studied and used extensively [20-28].

Recently, Li and Feng proposed a modified Liu-Storey conjugate gradient (MLS) method [24]. A good property of the MLS method is that, with any line search method, it can always generate descent search directions for objective functions. When the strong Wolfe line search is used, $\mathrm{Li}$ and Feng proved that their method is convergent globally.

The numerical algorithm for solving nonlinear systems of equations has been widely studied in the last decades. There are some numerical algorithms for nonlinear systems based on conjugate gradient methods [29-35]. Yuan and Zhang [29] proposed a numerical method for large-scale nonlinear systems of equations based on a three-term Polak-Ribière-Polyak conjugate gradient method and the hyperplane projection strategy given by Solodov and Svaiter [8]. Abubakar and Kumam [32] improved this three-term method by combining the idea of Yan et al. [36]. Yuan et al. [30] also extended the Hestenes-Stiefel conjugate gradient method to solve large-scale nonlinear equations and establish its global convergence. Bala and Kumam [31] proposed a numerical method for nonlinear systems by combining a descent Dai-Liao conjugate gradient and hyperplane projection method. Yu [34] extended the PRP method to solve large-scale nonlinear systems of equations based on the Grippo-Lampariello-Lucidi (GLL) [12] and Li-Fukushima (LF) [5] nonmonotone line search.

Inspired by the CG-type methods for the nonlinear system of equations, in this paper, we attempt to extend the MLS conjugate gradient method to solve problem (1) for the sufficient descent property, global convergence, and excellent numerical performance. To guarantee the global convergence of the new method, we determine the step length by a nonmonotone line search method which is a modification of the line search in $[14,15]$.

Our paper is organized as follows: in the second section, we introduce the algorithm. In Section 3, we analyze the global convergence of the proposed method. Some numerical experiment results are reported and analyzed in the last section.

Throughout this paper, we denote the Euclidean norm of vectors by $\|\cdot\|$, and $J(x)$ means the Jacobian matrix of $F$ at $x$.

\section{Algorithm}

In the remainder of this paper, we let

$$
f(x)=\frac{1}{2}\|F(x)\|^{2} .
$$

Based on the analysis in Section 1, we now extend the MLS method [24] to solve (1), and the steps of the algorithm are stated as follows.

$$
\sum_{k=0}^{\infty} \eta_{k}=\eta<\infty
$$

Step 1: compute $d_{k}$ by

$$
\begin{aligned}
d_{k} & =-F_{k}+\beta_{k}^{\mathrm{MLS}} d_{k-1}, \quad d_{0}=-F_{0}, \\
\beta_{k}^{\mathrm{MLS}} & =-\frac{F_{k}^{T} y_{k-1}}{F_{k-1}^{T} d_{k-1}}-t \frac{\left\|y_{k-1}\right\|^{2} F_{k}^{T} d_{k-1}}{\left(F_{k-1}^{T} d_{k-1}\right)^{2}},
\end{aligned}
$$

where $F_{k}=F\left(x_{k}\right),\left(y_{k-1}=F_{k}-F_{k-1}\right)$ and $t>(1 / 4)$ is a constant.

Step 2: select a suitable initial step length $\alpha_{k_{0}} \in\left[\alpha_{\min }\right.$, $\left.\alpha_{\text {max }}\right]$, and set $\alpha_{k}=\alpha_{k_{0}}$.

Step 3: nonmonotone line search step:

If

$$
f\left(x_{k}+\alpha_{k} d_{k}\right) \leq C_{k}+\eta_{k}-\lambda_{1} \alpha_{k}^{2}\left\|d_{k}\right\|^{4}-\lambda_{2} \alpha_{k}^{2} f\left(x_{k}\right),
$$

then set $x_{k+1}=x_{k}+\alpha_{k} d_{k}$, and go to Step 4 .

Else, if

$$
f\left(x_{k}-\alpha_{k} d_{k}\right) \leq C_{k}+\eta_{k}-\lambda_{1} \alpha_{k}^{2}\left\|d_{k}\right\|^{4}-\lambda_{2} \alpha_{k}^{2} f\left(x_{k}\right),
$$

then set $x_{k+1}=x_{k}-\alpha_{k} d_{k}$, and go to Step 4 .

Else, set $\left(\alpha_{k}=\rho \alpha_{k}\right)$, and go to Step 3 .

Step 4: stop if $\left\|F\left(x_{k+1}\right)\right\|=0$.

Step 5: select $\tau_{k} \in\left[\tau_{\min }, \tau_{\max }\right]$, and let

$$
\begin{aligned}
Q_{k+1} & =\tau_{k} Q_{k}+1, \\
C_{k+1} & =\frac{\tau_{k} Q_{k}\left(C_{k}+\eta_{k}\right)+f\left(x_{k+1}\right)}{Q_{k+1}} .
\end{aligned}
$$

Set $k=k+1$, and go to Step 1 .

The line search is the modification of the nonmonotone line search of Cheng and Li [14]. Define

$$
A_{k}=\frac{\sum_{i=0}^{k}\left(f\left(x_{i}\right)+i \eta_{i-1}\right)}{k+1}, \quad \eta_{-1}=0 .
$$

The following lemma shows that if $\tau_{k} \in[0,1]$, the parameter $C_{k}$ lies between $f_{k}$ and $A_{k}$.

Lemma 1. Suppose that $\left\{f\left(x_{k}\right)\right\}\left\{C_{k}\right\}$ and $\left\{A_{k}\right\}$ are infinite sequences generated by Algorithm 1. Then, $\left(f\left(x_{k}\right) \leq C_{k} \leq A_{k}\right)$ for all $k \geq 0$, and

$$
C_{k+1} \leq C_{k}+\eta_{k}
$$

We omit the proof of Lemma 1 since it is similar to Lemma 2.2 in [14]. It is clear that Step 3 will be finished in a finite number of iterations for $k=0$ since $C_{0}=f\left(x_{0}\right)$ and $f$ is continuously differentiable. Suppose that the line search step finishes in a finite number of iterations for index $k$. Then, we get from the line search conditions that 
Step 0: given $x_{0} \in R^{n}$, which is an initial guess of the solution. Let $0<\rho<1, \lambda_{1}, \lambda_{2}>0,0<\tau_{\min }<\tau_{\max }<1$, and $0<\alpha_{\min }<\alpha_{\text {max }}$ be given positive constants. Set $k=0, C_{0}=f\left(x_{0}\right)$, and $Q_{0}=1$. Select a positive sequence $\left\{\eta_{k}\right\}$ such that

Algorithm 1: Derivative-free MLS method:

$$
f\left(x_{k+1}\right) \leq C_{k}+\eta_{k}
$$

From the definition of $Q_{k}$ and $C_{k}$, we have

$$
\begin{aligned}
C_{k+1} & =\frac{\tau_{k} Q_{k}\left(C_{k}+\eta_{k}\right)+f\left(x_{k+1}\right)}{Q_{k+1}}, \\
& =\frac{\tau_{k} Q_{k}\left(C_{k}+\eta_{k}\right)+f\left(x_{k+1}\right)}{\tau_{k} Q_{k}+1}, \\
& >f\left(x_{k+1}\right) .
\end{aligned}
$$

This means condition (7) or (8) will be satisfied when $\alpha_{k}$ is small enough. Therefore, the algorithm is well defined.

The formula for direction $d_{k}$ is based on the MLS method proposed by Li and Feng [24]. From the definition of $d_{k}$, we have that if $F_{k} \neq 0$ and $F_{k-1}^{T} d_{k-1} \neq 0$ for some $k \geq 1$, then

$$
\begin{aligned}
F_{k}^{T} d_{k}= & -\left\|F_{k}\right\|^{2}+\beta_{k}^{\mathrm{MLS}} F_{k}^{T} d_{k-1}, \\
= & -\left\|F_{k}\right\|^{2}-\left(\frac{F_{k}^{T} y_{k-1}}{F_{k-1}^{T} d_{k-1}}+t \frac{\left\|y_{k-1}\right\|^{2} F_{k}^{T} d_{k-1}}{\left(F_{k-1}^{T} d_{k-1}\right)^{2}}\right) F_{k}^{T} d_{k-1}, \\
= & \frac{-\left\|F_{k}\right\|^{2}\left(F_{k-1}^{T} d_{k-1}\right)^{2}-\left(F_{k}^{T} y_{k-1}\right)\left(F_{k}^{T} d_{k-1}\right)\left(F_{k-1}^{T} d_{k-1}\right)-t\left\|y_{k-1}\right\|^{2}\left(F_{k}^{T} d_{k-1}\right)^{2}}{\left(F_{k-1}^{T} d_{k-1}\right)^{2}}, \\
\leq & \frac{-\left\|F_{k}\right\|^{2}\left(F_{k-1}^{T} d_{k-1}\right)^{2}+(1 / 2)(1 / 2 t)\left\|F_{k}\right\|^{2}\left(F_{k-1}^{T} d_{k-1}\right)^{2}+(1 / 2) 2 t\left\|y_{k-1}\right\|^{2}\left(F_{k}^{T} d_{k-1}\right)^{2}}{\left(F_{k-1}^{T} d_{k-1}\right)^{2}}, \\
& -\frac{t\left\|y_{k-1}\right\|^{2}\left(F_{k}^{T} d_{k-1}\right)^{2}}{\left(F_{k-1}^{T} d_{k-1}\right)^{2}}, \\
= & \left(\frac{1}{4 t}-1\right)\left\|F_{k}\right\|^{2},
\end{aligned}
$$

where the inequality follows from an upper bound for the second term of the third equation, which is obtained from $u^{T} v \leq(1 / 2)\left(\|u\|^{2}+\|v\|^{2}\right)$ with $u=(-1 / \sqrt{2 t})\left(F_{k-1}^{T} d_{k-1}\right) F_{k}$ and $v=\sqrt{2 t}\left(F_{k}^{T} d_{k-1}\right) y_{k-1}$. Obviously, inequality (14) also holds for $k=0$. Therefore, the search direction $d_{k}$ will always satisfy

$$
F_{k}^{T} d_{k} \leq\left(\frac{1}{4 t}-1\right)\left\|F_{k}\right\|^{2}<0
$$

This means that the scalar $\beta_{k}^{M L S}$ in (6) is well defined as long as $F_{k-1} \neq 0$ for $k \geq 1$ since $F_{k-1}^{T} d_{k-1} \neq 0$ by (15). Moreover, Algorithm 1 does not need to calculate the Jacobian matrix of $F$ and occupy low-memory space; therefore, it is suitable for large-scale problems. For convenience, we call this algorithm as the DF-MLS method.

\section{Convergence Property}

In this section, we devote to analyze the global convergence of our method under the following assumptions.

Assumption 1.

(i) The level set $\Omega=\left\{x \in R^{n} \mid f(x) \leq f\left(x_{0}\right)+\eta\right\}$ is bounded, where $x_{0} \in R^{n}$ is an arbitrary initial point and $\eta$ is a constant such that

$$
\sum_{k=0}^{\infty} \eta_{k}=\eta<\infty .
$$


(ii) The function $F(x)$ is continuously differentiable on some neighborhood $\Gamma$ of $\Omega$. Hence, $F(x)$ is Lipschitz continuous on $\Gamma$. That is, there exists a constant $L>0$ such that

$$
\|F(x)-F(y)\| \leq L\|x-y\|, \quad \forall x, y \in \Gamma .
$$

The assumption implies that there is a constant $\gamma_{1}>0$ such that

$$
\|F(x)\| \leq \gamma_{1}, \quad \forall x \in \Omega
$$

Lemma 2. Suppose that $\left\{x_{k}\right\}$ is an infinite sequence generated by the DF-MLS method and Assumption 1 holds. Then, $\left\{x_{k}\right\}$ is contained in $\Omega$.

Proof. Based on the line search step of the algorithm, we have

$$
f\left(x_{k}\right) \leq C_{k}+\eta_{k}, \quad \forall k \geq 0 .
$$

Combining with (11), we have

$$
\begin{aligned}
f\left(x_{k}\right) & \leq C_{k}+\eta_{k} \leq C_{k-1}+\eta_{k-1}+\eta_{k} \\
& \leq \cdots \leq f\left(x_{0}\right)+\sum_{i=0}^{k} \quad \eta_{k} \leq f\left(x_{0}\right)+\eta .
\end{aligned}
$$

The above inequality draws the conclusion.

Lemma 3. Suppose that $\left\{x_{k}\right\}$ is an infinite sequence generated by the DF-MLS method and Assumption 1 holds. Then,

$$
\begin{aligned}
& \lim _{k \longrightarrow \infty} \alpha_{k}\left\|d_{k}\right\|^{2}=0, \\
& \lim _{k \longrightarrow \infty} \alpha_{k}^{2} f\left(x_{k}\right)=0 .
\end{aligned}
$$

Proof. We get from the third step of the algorithm that

$$
f\left(x_{k+1}\right) \leq C_{k}+\eta_{k}-\lambda_{1} \alpha_{k}^{2}\left\|d_{k}\right\|^{4}-\lambda_{2} \alpha_{k}^{2} f\left(x_{k}\right) .
$$

Combining this with (9), we have

$$
\begin{aligned}
C_{k+1} & =\frac{\tau_{k} Q_{k}\left(C_{k}+\eta_{k}\right)+f\left(x_{k+1}\right)}{Q_{k+1}}, \\
& \leq \frac{\tau_{k} Q_{k}\left(C_{k}+\eta_{k}\right)+C_{k}+\eta_{k}-\lambda_{1} \alpha_{k}^{2}\left\|d_{k}\right\|^{4}-\lambda_{2} \alpha_{k}^{2} f\left(x_{k}\right)}{Q_{k+1}}, \\
& =C_{k}+\eta_{k}-\frac{\lambda_{1} \alpha_{k}^{2}\left\|d_{k}\right\|^{4}+\lambda_{2} \alpha_{k}^{2} f\left(x_{k}\right)}{Q_{k+1}} .
\end{aligned}
$$

By (4), we have

$$
\sum_{k=0}^{\infty} \frac{\lambda_{1} \alpha_{k}^{2}\left\|d_{k}\right\|^{4}+\lambda_{2} \alpha_{k}^{2} f\left(x_{k}\right)}{Q_{k+1}}<\infty .
$$

Since $0<\tau_{\min } \leq \tau_{k} \leq \tau_{\max }<1$ and $Q_{0}=1$, we get from (9) that

$$
Q_{k+1}=\tau_{k} Q_{k}+1=1+\sum_{j=0}^{k} \prod_{i=0}^{j} \tau_{k-i} \leq 1+\sum_{j=0}^{k} \tau_{\max }^{j+1} \leq \frac{1}{1-\tau_{\max }} .
$$

This together with (24) implies

$$
\sum_{k=0}^{\infty} \lambda_{1} \alpha_{k}^{2}\left\|d_{k}\right\|^{4}+\lambda_{2} \alpha_{k}^{2} f\left(x_{k}\right)<\infty,
$$

which gives (21). This completes the proof.

Lemma 4. Suppose that $\left\{d_{k}\right\}$ is an infinite sequence generated by the DF-MLS method and Assumption 1 holds. If there exists a constant $\gamma>0$ such that

$$
\left\|F_{k}\right\| \geq \gamma, \quad \forall k \geq 0
$$

then there exists a constant $B>0$ such that

$$
\begin{gathered}
\left\|d_{k}\right\| \leq B, \quad \forall k \geq 0, \\
\lim _{k \rightarrow \infty} \beta_{k}^{\mathrm{MLS}}\left\|d_{k-1}\right\|=0 .
\end{gathered}
$$

Proof. We get from (15), (17), (18), and (5) that

$$
\begin{aligned}
\left\|d_{k}\right\| \leq & \left\|F_{k}\right\|+\left|\beta_{k}^{\mathrm{MLS}}\right|\left\|d_{k-1}\right\|, \\
\leq & \left\|F_{k}\right\|+\frac{\left\|F_{k}\right\|\left\|y_{k-1}\right\|\left\|d_{k-1}\right\|}{\left|F_{k-1}^{T} d_{k-1}\right|}+t \frac{\left\|y_{k-1}\right\|^{2}\left\|F_{k}\right\|\left\|d_{k-1}\right\|}{\left|F_{k-1}^{T} d_{k-1}\right|^{2}}\left\|d_{k-1}\right\|, \\
\leq & \gamma_{1}+\frac{4 t \gamma_{1} L \alpha_{k-1}\left\|d_{k-1}\right\|^{2}}{(4 t-1) \gamma^{2}}+\frac{t(4 t)^{2} 2 \gamma_{1}^{2} L \alpha_{k-1}\left\|d_{k-1}\right\|^{2}}{(4 t-1)^{2} \gamma^{4}}\left\|d_{k-1}\right\|, \\
= & \gamma_{1}+\frac{4 t \gamma_{1} L}{(4 t-1) \gamma^{2}} \alpha_{k-1}\left\|d_{k-1}\right\|^{2} \\
& +\frac{32 t^{3} \gamma_{1}^{2} L}{(4 t-1)^{2} \gamma^{4}} \alpha_{k-1}\left\|d_{k-1}\right\|^{2}\left\|d_{k-1}\right\| .
\end{aligned}
$$

For any constant $b \in(0,1)$, we get from (21) that there exists an index $k_{0}$ such that

$$
\frac{32 t^{3} \gamma_{1}^{2} L}{(4 t-1)^{2} \gamma^{4}} \alpha_{k-1}\left\|d_{k-1}\right\|^{2}<b, \quad \forall k>k_{0} .
$$

Then,

$$
\left\|d_{k}\right\| \leq \gamma_{1}+\frac{(4 t-1) \gamma^{2} b}{8 t^{2} \gamma_{1}}+b\left\|d_{k-1}\right\|=c+b\left\|d_{k-1}\right\|,
$$

where $c=\gamma_{1}+\left((4 t-1) \gamma^{2} b /\left(8 t^{2} \gamma_{1}\right)\right)$ is a constant. For any $k>k_{0}$, we have 


$$
\left\|d_{k}\right\| \leq c\left(1+b+b^{2}+\cdots+b^{k-k_{0}+1}\right)+b^{k-k_{0}}\left\|d_{k_{0}}\right\| \leq \frac{c}{1-b}+\left\|d_{k_{0}}\right\| .
$$

So, we can let $B=\max \left\{\left\|d_{1}\right\|,\left\|d_{2}\right\|, \ldots,\left\|d_{k_{0}}\right\|, c / 1-b+\right.$ $\left.\left\|d_{k_{0}}\right\|\right\}$ to get (28). By (17), (27), (21), and (28), we have

$\left|\beta_{k}^{\mathrm{MLS}}\right|\left\|d_{k-1}||=\left|\beta_{k}^{\mathrm{MLS}}\right|\right\| d_{k-1} \|$,

$$
\begin{aligned}
& \leq \frac{\left\|F_{k}\right\|\left\|y_{k-1}\right\|\left\|d_{k-1}\right\|}{\left|F_{k-1}^{T} d_{k-1}\right|}+t \frac{\left\|y_{k-1}\right\|^{2}\left\|F_{k}\right\|\left\|d_{k-1}\right\|}{\left|F_{k-1}^{T} d_{k-1}\right|^{2}}\left\|d_{k-1}\right\|, \\
& \leq\left(\frac{4 t \gamma_{1} L}{(4 t-1) \gamma^{2}}+\frac{32 t^{3} \gamma_{1}^{2} L}{(4 t-1)^{2} \gamma^{4}}\left\|d_{k-1}\right\|\right) \alpha_{k-1}\left\|d_{k-1}\right\|^{2},
\end{aligned}
$$

which shows (29).

Analogy to Theorem 1 in [13], we establish the global convergence of the DF-MLS method as follows.

Theorem 1. Suppose that $\left\{x_{k}\right\}$ is an infinite sequence generated by the DF-MLS method and Assumption 1 holds. Then,

$$
\underset{k \longrightarrow \infty}{\liminf }\left\|F_{k}\right\|=0
$$

or every limit point $x^{*}$ of $\left\{x_{k}\right\}$ satisfies

$$
F\left(x^{*}\right)^{T} J\left(x^{*}\right) F\left(x^{*}\right)=0,
$$

where $J\left(x^{*}\right)$ is the Jacobian matrix of $F(x)$ at $x^{*}$.

Proof. Let $x^{*}$ be a limit point of $\left\{x_{k}\right\}$ and $\mathbb{K}$ be an infinite sequence of indices such that

$$
\lim _{k \in \mathbb{K}} x_{k}=x^{*} \text {. }
$$

(21) implies

$$
\lim _{k \in \mathbb{K}} \alpha_{k}^{2} f\left(x_{k}\right)=\lim _{k \in \mathbb{K}} \frac{1}{2} \alpha_{k}^{2}\left\|F_{k}\right\|^{2}=0 .
$$

If $\lim \alpha_{k} \neq 0$, then there exists an infinite subsequence $\mathbb{K}_{1} \subseteq \mathbb{K}^{k \in \mathbb{S}}$ such that $\alpha_{k}$ is bounded away from zero for any $k \in \mathbb{K}_{1}$. Therefore, in this case, we get from (38) that

$$
\lim _{k \in \mathbb{K}_{1}}\left\|F_{k}\right\|=0
$$

This implies $\left\|F\left(x^{*}\right)\right\|=0$, and (35) holds since $F$ is continuous and $\lim _{k \in \mathbb{K}_{1}} x_{k}=x^{*}$.

Next, we analyze the case when

$$
\lim _{k \in \mathbb{K}} \alpha_{k}=0 .
$$

Assume that (35) does not hold; then, there exists an infinite subsequence $\mathbb{K}_{2} \subseteq \mathbb{K}$ such that $\left\|F_{k}\right\|$ is bounded away from zero for any $k \in \mathbb{K}_{2}$. From the third step of the DF-MLS method, the step length $\rho^{-1} \alpha_{k}$ will satisfy neither (7) nor (8) when $k \in \mathbb{K}_{2}$ is large enough. This means $f\left(x_{k}+\rho^{-1} \alpha_{k} d_{k}\right)>C_{k}+\eta_{k}-\lambda_{1} \rho^{-2} \alpha_{k}^{2}\left\|d_{k}\right\|^{4}-\lambda_{2} \rho^{-2} \alpha_{k}^{2} f\left(x_{k}\right)$,

$f\left(x_{k}-\rho^{-1} \alpha_{k} d_{k}\right)>C_{k}+\eta_{k}-\lambda_{1} \rho^{-2} \alpha_{k}^{2}\left\|d_{k}\right\|^{4}-\lambda_{2} \rho^{-2} \alpha_{k}^{2} f\left(x_{k}\right)$.

We get from the conclusion of Lemma 1 that $C_{k} \geq f\left(x_{k}\right) \geq 0$. Combining this with (41), we have

$$
f\left(x_{k}+\rho^{-1} \alpha_{k} d_{k}\right)>f\left(x_{k}\right)-\lambda_{1} \rho^{-2} \alpha_{k}^{2}\left\|d_{k}\right\|^{4}-\lambda_{2} \rho^{-2} \alpha_{k}^{2} f\left(x_{k}\right) \text {. }
$$

It follows from Lemma 2 that

$$
f\left(x_{k}\right) \leq f\left(x_{0}\right)+\eta \text {. }
$$

Combining this with (18) and (28), we obtain

$$
f\left(x_{k}+\rho^{-1} \alpha_{k} d_{k}\right)-f\left(x_{k}\right)>-M \alpha_{k}^{2},
$$

where $M=\lambda_{1} \rho^{-2} B^{4}+\lambda_{2} \rho^{-2}\left(f\left(x_{0}\right)+\eta\right)$. Therefore,

$$
\frac{f\left(x_{k}+\rho^{-1} \alpha_{k} d_{k}\right)-f\left(x_{k}\right)}{\alpha_{k}}>-M \alpha_{k} .
$$

By the mean value theorem and (3), there exists a parameter $\xi_{k} \in(0,1)$ such that

$$
\rho^{-1} F\left(x_{k}+\xi_{k} \rho^{-1} \alpha_{k} d_{k}\right)^{T} J\left(x_{k}+\xi_{k} \rho^{-1} \alpha_{k} d_{k}\right) d_{k}>-M \alpha_{k},
$$

namely,

$$
\begin{aligned}
& \rho^{-1} F\left(x_{k}+\xi_{k} \rho^{-1} \alpha_{k} d_{k}\right)^{T} J\left(x_{k}+\xi_{k} \rho^{-1} \alpha_{k} d_{k}\right)\left(-F_{k}+\beta_{k}^{\mathrm{MLS}} d_{k-1}\right) \\
& \quad>-M \alpha_{k} .
\end{aligned}
$$

Combining this with (21), (29), and (40) and taking limits in (48), we obtain

$$
F\left(x^{*}\right)^{T} J\left(x^{*}\right) F\left(x^{*}\right) \leq 0 .
$$

Using (42) and proceeding in a similar way, we can get

$$
F\left(x^{*}\right)^{T} J\left(x^{*}\right) F\left(x^{*}\right) \geq 0 .
$$

From the last two inequalities, we get (36) which completes the proof.

Corollary 1. Suppose that $\left\{x_{k}\right\}$ is an infinite sequence generated by the DF-MLS method and Assumption 1 holds. If $x^{*}$ is a limit point of $\left\{x_{k}\right\}$ and

$$
y^{T} J\left(x^{*}\right) y \neq 0, \quad \forall y \in \mathbb{R}^{n}, y \neq 0,
$$

then

$$
F\left(x^{*}\right)=0 .
$$


TABLe 1: Numerical results.

\begin{tabular}{|c|c|c|c|c|c|}
\hline Prob & Dim & $\begin{array}{c}\text { DF-SANE } \\
\text { Iter/Nfun/Time }\end{array}$ & $\begin{array}{l}\text { N-DF-SANE } \\
\text { Iter/Nfun/Time }\end{array}$ & $\begin{array}{l}\text { DF-SCGNE } \\
\text { Iter/Nfun/Time }\end{array}$ & $\begin{array}{c}\text { DF-MLS } \\
\text { Iter/Nfun/Time }\end{array}$ \\
\hline ARGLINA & 50 & $3 / 3 / 0.001$ & $3 / 5 / 0.001$ & $3 / 6 / 0.001$ & $2 / 5 / 0.001$ \\
\hline ARGLINA & 200 & $3 / 3 / 0.005$ & $3 / 5 / 0.007$ & $3 / 6 / 0.009$ & $2 / 5 / 0.008$ \\
\hline ARGLINB & 100 & $47 / 1151 / 0.298$ & $3 / 29 / 0.008$ & $3 / 30 / 0.008$ & $31 / 817 / 0.209$ \\
\hline ARGLINB & 200 & $867 / 24347 / 25.89$ & $3 / 33 / 0.039$ & $4 / 35 / 0.041$ & $31 / 923 / 0.988$ \\
\hline ARGLINC & 100 & $231 / 5707 / 1.441$ & $3 / 29 / 0.008$ & $3 / 30 / 0.009$ & $33 / 865 / 0.216$ \\
\hline ARGLINC & 200 & $39 / 1071 / 1.133$ & $3 / 33 / 0.037$ & $4 / 35 / 0.04$ & $34 / 1023 / 1.071$ \\
\hline ARWHEAD & 500 & $7 / 15 / 0.002$ & $5 / 17 / 0.003$ & $5 / 17 / 0.003$ & $5 / 17 / 0.002$ \\
\hline ARWHEAD & 5000 & $6 / 16 / 0.021$ & $5 / 19 / 0.027$ & $5 / 19 / 0.026$ & $5 / 19 / 0.025$ \\
\hline BDQRTIC & 500 & $67 / 131 / 0.02$ & $86 / 297 / 0.046$ & $21 / 52 / 0.009$ & $16 / 41 / 0.006$ \\
\hline BDQRTIC & 5000 & $50 / 110 / 0.192$ & $194 / 1019 / 1.758$ & $16 / 45 / 0.083$ & $2 / 35 / 0.062$ \\
\hline $\mathrm{BOX}$ & 100 & $66 / 198 / 0.006$ & $309 / 873 / 0.028$ & $78 / 372 / 0.012$ & $37 / 153 / 0.005$ \\
\hline BOX & 1000 & - & $706 / 4367 / 1.344$ & $124 / 743 / 0.23$ & $157 / 1255 / 0.384$ \\
\hline BROWNAL & 100 & $3 / 13 / 0.002$ & $3 / 15 / 0.002$ & $3 / 15 / 0.002$ & $3 / 15 / 0.002$ \\
\hline BROYDN7D & 1000 & - & $68 / 137 / 0.138$ & $24 / 49 / 0.049$ & $25 / 51 / 0.051$ \\
\hline BRYBND & 1000 & $18 / 29 / 0.012$ & $39 / 85 / 0.036$ & $102 / 388 / 0.16$ & $13 / 31 / 0.013$ \\
\hline BRYBND & 5000 & $23 / 33 / 0.08$ & $36 / 75 / 0.186$ & $17 / 37 / 0.093$ & $16 / 37 / 0.089$ \\
\hline CHAINWOO & 4000 & $170 / 355 / 0.431$ & $94 / 332 / 0.414$ & $105 / 476 / 0.586$ & $64 / 201 / 0.247$ \\
\hline CHAINWOO & 10000 & $41 / 67 / 0.235$ & - & $49 / 178 / 0.605$ & $22 / 51 / 0.175$ \\
\hline CHNROSNB & 50 & - & - & - & - \\
\hline CRAGGLVY & 500 & $28 / 44 / 0.015$ & $35 / 81 / 0.025$ & $19 / 45 / 0.016$ & $19 / 45 / 0.015$ \\
\hline CRAGGLVY & 1000 & $32 / 50 / 0.032$ & $43 / 125 / 0.072$ & $22 / 51 / 0.034$ & $18 / 43 / 0.029$ \\
\hline CURLY10 & 1000 & - & $185 / 752 / 0.161$ & $100 / 216 / 0.05$ & $118 / 357 / 0.074$ \\
\hline CURLY20 & 1000 & - & $182 / 942 / 0.324$ & $121 / 475 / 0.168$ & $182 / 919 / 0.313$ \\
\hline CURLY30 & 100 & - & $809 / 4720 / 0.198$ & $621 / 4448 / 0.187$ & $757 / 5431 / 0.225$ \\
\hline DECONVU & 61 & $19 / 23 / 0.001$ & $24 / 51 / 0.002$ & $27 / 57 / 0.002$ & $22 / 47 / 0.002$ \\
\hline DIXMAANA & 1500 & $6 / 8 / 0.003$ & $5 / 11 / 0.004$ & $6 / 13 / 0.005$ & $5 / 11 / 0.004$ \\
\hline DIXMAANA & 9000 & $6 / 8 / 0.021$ & $5 / 11 / 0.029$ & $6 / 13 / 0.036$ & $7 / 17 / 0.042$ \\
\hline DIXMAANB & 3000 & $7 / 9 / 0.007$ & $6 / 13 / 0.01$ & $10 / 21 / 0.017$ & $7 / 17 / 0.014$ \\
\hline DIXMAANB & 9000 & $7 / 9 / 0.023$ & $6 / 13 / 0.035$ & $10 / 21 / 0.054$ & $7 / 17 / 0.043$ \\
\hline DIXMAANC & 3000 & $9 / 13 / 0.011$ & $7 / 17 / 0.013$ & $9 / 21 / 0.017$ & $7 / 17 / 0.013$ \\
\hline DIXMAANC & 9000 & $9 / 13 / 0.032$ & $7 / 17 / 0.046$ & $9 / 21 / 0.058$ & $7 / 17 / 0.042$ \\
\hline DIXMAAND & 1500 & $7 / 11 / 0.004$ & $6 / 15 / 0.005$ & $7 / 17 / 0.009$ & $5 / 13 / 0.005$ \\
\hline DIXMAAND & 3000 & $7 / 11 / 0.008$ & $6 / 15 / 0.013$ & $7 / 17 / 0.014$ & $5 / 13 / 0.01$ \\
\hline DIXMAANE & 3000 & $22 / 26 / 0.019$ & $47 / 95 / 0.075$ & $20 / 41 / 0.032$ & $26 / 55 / 0.041$ \\
\hline DIXMAANE & 9000 & $22 / 26 / 0.064$ & $47 / 95 / 0.244$ & $20 / 41 / 0.105$ & $26 / 55 / 0.138$ \\
\hline DIXMAANF & 3000 & $17 / 19 / 0.015$ & $25 / 51 / 0.041$ & $17 / 35 / 0.028$ & $13 / 29 / 0.022$ \\
\hline DIXMAANF & 9000 & $17 / 19 / 0.047$ & $25 / 51 / 0.13$ & $17 / 35 / 0.09$ & $13 / 29 / 0.073$ \\
\hline DIXMAANG & 3000 & $14 / 18 / 0.014$ & $13 / 29 / 0.023$ & $12 / 27 / 0.021$ & $11 / 25 / 0.019$ \\
\hline DIXMAANG & 9000 & $14 / 18 / 0.044$ & $13 / 29 / 0.076$ & $12 / 27 / 0.07$ & $11 / 25 / 0.062$ \\
\hline DIXMAANH & 3000 & 9/13/0.01 & $9 / 21 / 0.017$ & $9 / 21 / 0.017$ & $8 / 19 / 0.014$ \\
\hline DIXMAANH & 9000 & $9 / 13 / 0.034$ & $9 / 21 / 0.054$ & $9 / 21 / 0.054$ & $11 / 27 / 0.067$ \\
\hline DIXMAANI & 3000 & $29 / 37 / 0.028$ & $84 / 169 / 0.133$ & $29 / 59 / 0.048$ & $37 / 77 / 0.058$ \\
\hline DIXMAANI & 9000 & $29 / 37 / 0.093$ & $84 / 169 / 0.4319$ & $29 / 59 / 0.15$ & $37 / 77 / 0.19$ \\
\hline DIXMAANJ & 3000 & $18 / 20 / 0.016$ & $33 / 67 / 0.054$ & $23 / 47 / 0.037$ & $15 / 33 / 0.025$ \\
\hline DIXMAANJ & 9000 & $18 / 20 / 0.051$ & $33 / 67 / 0.172$ & $23 / 47 / 0.121$ & $15 / 33 / 0.083$ \\
\hline DIXMAANK & 1500 & $14 / 18 / 0.006$ & $15 / 33 / 0.013$ & $14 / 31 / 0.011$ & $12 / 27 / 0.01$ \\
\hline DIXMAANK & 3000 & $14 / 18 / 0.013$ & $15 / 33 / 0.027$ & $14 / 31 / 0.025$ & $12 / 27 / 0.021$ \\
\hline DIXMAANL & 1500 & $12 / 16 / 0.006$ & $9 / 21 / 0.009$ & $9 / 21 / 0.007$ & $8 / 19 / 0.007$ \\
\hline DIXMAANL & 3000 & $12 / 16 / 0.012$ & $9 / 21 / 0.018$ & $9 / 21 / 0.019$ & $8 / 19 / 0.014$ \\
\hline DIXON3DQ & 10000 & - & - & $609 / 1235 / 1.459$ & - \\
\hline DQDRTIC & 500 & $12 / 18 / 0.001$ & $56 / 175 / 0.017$ & $7 / 19 / 0.001$ & $7 / 19 / 0.002$ \\
\hline DQDRTIC & 1000 & $12 / 18 / 0.003$ & $40 / 91 / 0.02$ & $7 / 19 / 0.004$ & $7 / 19 / 0.004$ \\
\hline EDENSCH & 2000 & $19 / 25 / 0.014$ & $12 / 29 / 0.018$ & $17 / 39 / 0.023$ & $13 / 33 / 0.019$ \\
\hline EG2 & 1000 & $9 / 15 / 0.004$ & $6 / 13 / 0.004$ & $16 / 37 / 0.011$ & $6 / 13 / 0.006$ \\
\hline ENGVAL1 & 100 & $12 / 16 / 0.001$ & $14 / 31 / 0.001$ & $9 / 21 / 0.001$ & $9 / 21 / 0.001$ \\
\hline ENGVAL1 & 5000 & $8 / 12 / 0.016$ & $7 / 17 / 0.025$ & $9 / 21 / 0.03$ & $6 / 15 / 0.02$ \\
\hline ERRINROS & 50 & $20 / 32 / 0.001$ & $12 / 33 / 0.001$ & $16 / 41 / 0.001$ & $12 / 33 / 0.001$ \\
\hline EXTROSNB & 100 & $40 / 55 / 0.001$ & $36 / 81 / 0.001$ & $19 / 44 / 0.001$ & $17 / 43 / 0$ \\
\hline EXTROSNB & 1000 & $22 / 28 / 0.004$ & $17 / 39 / 0.007$ & $6 / 19 / 0.003$ & $18 / 51 / 0.008$ \\
\hline
\end{tabular}


TABle 1: Continued.

\begin{tabular}{|c|c|c|c|c|c|}
\hline Prob & Dim & $\begin{array}{c}\text { DF-SANE } \\
\text { Iter/Nfun/Time }\end{array}$ & $\begin{array}{c}\text { N-DF-SANE } \\
\text { Iter/Nfun/Time }\end{array}$ & $\begin{array}{c}\text { DF-SCGNE } \\
\text { Iter/Nfun/Time }\end{array}$ & $\begin{array}{c}\text { DF-MLS } \\
\text { Iter/Nfun/Time }\end{array}$ \\
\hline FLETCBV2 & 10000 & $1 / 1 / 0.005$ & $1 / 1 / 0.006$ & $1 / 1 / 0.005$ & $1 / 1 / 0.005$ \\
\hline FLETCBV3 & 100 & - & - & - & - \\
\hline FREUROTH & 1000 & $816 / 3457 / 1.068$ & $78 / 266 / 0.085$ & $64 / 235 / 0.074$ & $37 / 97 / 0.031$ \\
\hline GENHUMPS & 5000 & - & - & - & - \\
\hline GENROSE & 500 & - & - & - & - \\
\hline HILBERTA & 10 & $12 / 12 / 0$ & $93 / 193 / 0.001$ & $7 / 14 / 0$ & $6 / 11 / 0.001$ \\
\hline HILBERTB & 50 & $5 / 7 / 0.001$ & $5 / 11 / 0.002$ & $6 / 13 / 0.002$ & $5 / 11 / 0.001$ \\
\hline HYDC20LS & 99 & $777 / 3215 / 0.543$ & $342 / 1701 / 0.264$ & $314 / 1874 / 0.291$ & $107 / 447 / 0.069$ \\
\hline INDEF & 100 & - & - & - & - \\
\hline LIARWHD & 5000 & 43/97/0.109 & $957 / 7417 / 8.453$ & $35 / 94 / 0.116$ & $16 / 43 / 0.052$ \\
\hline LIARWHD & 10000 & - & - & $419 / 3534 / 8.681$ & $22 / 59 / 0.153$ \\
\hline MANCINO & 100 & $5 / 19 / 0.118$ & $5 / 23 / 0.146$ & $5 / 23 / 0.145$ & $63 / 351 / 2.1817$ \\
\hline MODBEALE & 2000 & $587 / 2400 / 2.926$ & $224 / 1261 / 1.576$ & $55 / 183 / 0.235$ & $31 / 78 / 0.098$ \\
\hline MOREBV & 1000 & $20 / 22 / 0.005$ & $49 / 97 / 0.021$ & $21 / 42 / 0.01$ & $14 / 27 / 0.005$ \\
\hline MOREBV & 5000 & $5 / 5 / 0.006$ & $6 / 11 / 0.012$ & $19 / 38 / 0.043$ & $3 / 5 / 0.006$ \\
\hline MSQRTALS & 1024 & - & - & - & - \\
\hline NONCVXUN & 100 & $37 / 45 / 0.002$ & $42 / 97 / 0.004$ & $30 / 63 / 0.003$ & $39 / 101 / 0.004$ \\
\hline NONDIA & 5000 & $3 / 15 / 0.016$ & $3 / 17 / 0.019$ & $4 / 19 / 0.022$ & $3 / 17 / 0.018$ \\
\hline NONDIA & 10000 & $4 / 18 / 0.041$ & $4 / 21 / 0.052$ & $4 / 21 / 0.05$ & $4 / 21 / 0.048$ \\
\hline NONDQUAR & 1000 & $24 / 36 / 0.004$ & $22 / 51 / 0.008$ & $30 / 69 / 0.009$ & $23 / 55 / 0.007$ \\
\hline NONDQUAR & 10000 & $15 / 27 / 0.034$ & $17 / 45 / 0.061$ & $30 / 73 / 0.098$ & $11 / 33 / 0.042$ \\
\hline NONMSQRT & 100 & - & $177 / 805 / 0.059$ & $80 / 285 / 0.022$ & $483 / 2815 / 0.198$ \\
\hline OSCIPATH & 500 & $11 / 13 / 0.001$ & $12 / 25 / 0.002$ & $10 / 22 / 0.002$ & 8/17/0.001 \\
\hline PENALTY1 & 50 & $10 / 20 / 0$ & $9 / 27 / 0.001$ & $9 / 28 / 0$ & $10 / 31 / 0$ \\
\hline PENALTY2 & 200 & $38 / 72 / 0.026$ & $58 / 181 / 0.056$ & $33 / 79 / 0.03$ & 29/71/0.026 \\
\hline POWELLSG & 5000 & $48 / 64 / 0.035$ & $251 / 591 / 0.336$ & $36 / 77 / 0.046$ & $24 / 53 / 0.03$ \\
\hline POWELLSG & 10000 & $48 / 64 / 0.073$ & $159 / 685 / 0.737$ & $36 / 77 / 0.096$ & $24 / 53 / 0.062$ \\
\hline POWER & 10000 & $15 / 321 / 0.26$ & $9 / 41 / 0.039$ & $10 / 43 / 0.042$ & $22 / 517 / 0.413$ \\
\hline SCHMVETT & 10000 & - & - & - & $39 / 96 / 0.930$ \\
\hline SCOSINE & 100 & - & - & - & - \\
\hline SENSORS & 100 & $439 / 598 / 4.047$ & $13 / 33 / 0.209$ & $17 / 39 / 0.246$ & $38 / 103 / 0.650$ \\
\hline SINQUAD & 5000 & - & $305 / 2441 / 5.297$ & - & $27 / 83 / 0.175$ \\
\hline SPARSQUR & 1000 & $13 / 21 / 0.008$ & $9 / 25 / 0.01$ & $14 / 35 / 0.014$ & 9/25/0.01 \\
\hline SPARSQUR & 5000 & $13 / 23 / 0.049$ & $10 / 29 / 0.066$ & $12 / 33 / 0.073$ & $10 / 29 / 0.061$ \\
\hline SPMSRTLS & 10000 & - & - & - & - \\
\hline SROSENBR & 1000 & $12 / 20 / 0.003$ & $120 / 637 / 0.077$ & $6 / 17 / 0.002$ & $9 / 23 / 0.003$ \\
\hline SROSENBR & 5000 & $12 / 20 / 0.012$ & $82 / 345 / 0.223$ & $6 / 17 / 0.012$ & $9 / 23 / 0.015$ \\
\hline TESTQUAD & 1000 & $43 / 63 / 0.006$ & $154 / 319 / 0.039$ & $38 / 87 / 0.009$ & $39 / 89 / 0.008$ \\
\hline TESTQUAD & 5000 & $43 / 65 / 0.03$ & $159 / 329 / 0.198$ & $43 / 97 / 0.053$ & $43 / 97 / 0.05$ \\
\hline TOINTGOR & 50 & $171 / 319 / 0.005$ & $130 / 433 / 0.007$ & $62 / 129 / 0.002$ & $65 / 145 / 0.003$ \\
\hline TOINTGSS & 1000 & $2 / 4 / 0.002$ & $3 / 7 / 0.004$ & 4/9/0.005 & $3 / 7 / 0.004$ \\
\hline TOINTPSP & 50 & $642 / 1771 / 0.015$ & $198 / 836 / 0.007$ & $162 / 676 / 0.006$ & $220 / 880 / 0.007$ \\
\hline TOINTQOR & 50 & $26 / 30 / 0.001$ & $87 / 177 / 0.002$ & $22 / 47 / 0.001$ & $22 / 47 / 0$ \\
\hline TQUARTIC & 1000 & - & $538 / 4539 / 0.713$ & - & $416 / 4104 / 0.642$ \\
\hline TQUARTIC & 10000 & - & - & - & - \\
\hline TRIDIA & 5000 & $655 / 2455 / 1.118$ & $390 / 2181 / 1.075$ & $206 / 419 / 0.245$ & $195 / 397 / 0.216$ \\
\hline TRIDIA & 10000 & $724 / 2890 / 2.760$ & $395 / 2219 / 2.251$ & $205 / 417 / 0.522$ & $205 / 419 / 0.484$ \\
\hline VARDIM & 100 & $5 / 103 / 0.001$ & $9 / 43 / 0.001$ & $10 / 45 / 0.001$ & $4 / 83 / 0.001$ \\
\hline VARDIM & 200 & $16 / 426 / 0.009$ & $10 / 49 / 0.001$ & $12 / 54 / 0.001$ & $7 / 187 / 0.004$ \\
\hline VAREIGVL & 1000 & $26 / 42 / 0.018$ & $27 / 59 / 0.028$ & $17 / 39 / 0.019$ & $19 / 43 / 0.019$ \\
\hline VAREIGVL & 5000 & $116 / 290 / 0.745$ & $124 / 371 / 1.000$ & $42 / 187 / 0.487$ & $36 / 117 / 0.303$ \\
\hline WOODS & 4000 & $31 / 43 / 0.033$ & $37 / 81 / 0.06$ & $42 / 117 / 0.083$ & $14 / 37 / 0.025$ \\
\hline WOODS & 10000 & $31 / 43 / 0.081$ & $37 / 81 / 0.163$ & $40 / 113 / 0.218$ & $14 / 37 / 0.071$ \\
\hline
\end{tabular}

Proof. We draw the conclusion straightforwardly by using Theorem 1.

If the Jacobian matrix of $F(x)$ is positive definite for all $x \in \mathbb{R}^{n}$, we say that the mapping $F$ is strictly monotone, namely, the Jacobian matrix $J(x)$ satisfies

$$
x^{T} J(x) x>0, \quad \forall x \in \mathbb{R}^{n}
$$

If mapping $F(x)$ is strictly monotone and $F(x)=0$ has a solution, its solution must be unique. See Chapter 5 of [37]. 
Corollary 2. Suppose that $\left\{x_{k}\right\}$ is an infinite sequence generated by the DF-MLS method and Assumption 1 holds. If $F$ or $-F$ is strictly monotone, then every bounded subsequence $\left\{x_{k}\right\}_{k \in \mathbb{N}}$ converges to the solution of (1).

Proof. We draw the conclusion straightforwardly by using Theorem 1.

\section{Numerical Results}

In this section, we report the numerical experimental results and analyze the efficiency of our method. We compare the numerical performance of our method with some other methods such as the DF-SANE method [13], the N-DFSANE method [14], and the DF-SCGNE method [34]. We used the aforementioned methods to find the stationary points of the test problems by using their gradients only. We tested 112 unconstrained optimization problems in the CUTEr library [38] with dimensions varying from 50 to 10,000 . We ran two versions of some problems for which the dimensions can be chosen randomly.

We downloaded the DF-SANE code from Professor Raydan's home page at http://kuainasi.ciens.ucv.ve/ mraydan/mraydan_pub.html. These four methods were coded in Fortran 77 and carried out on a personal computer with $2.8 \mathrm{GHz} \mathrm{CPU}$ processor and $2 \mathrm{~GB}$ RAM and Linux operating system. We terminated the program when

$$
\frac{\left\|F\left(x_{k}\right)\right\|}{\sqrt{n}} \leq e_{a}+e_{r} \frac{\left\|F\left(x_{0}\right)\right\|}{\sqrt{n}},
$$

where $e_{a}=10^{-5}$ and $e_{r}=10^{-4}$.

Table 1 lists the detailed experimental results, which include the problem names (Prob), dimensions (Dim), the total number of iterations (Iter), function evaluations (Nfun), and the CPU time (Time) in seconds, respectively. The symbol "-" means that the number of iterations exceeds 1000 , or the line search iterates more than 100 times. In other words, this means that the method failed to solve that problem.

We compared the numerical performance of the methods by using the profiles designed by Dolan and Moré [39]. Figures 1 and 2 describe the numerical performance of the methods relative to the CPU time (in seconds), the total number of iterations, and function value evaluations, respectively. The curves in the figures have the following meanings.

(1) "DF-SANE" denotes the DF-SANE method proposed by La Cruz et al. [13] with nexp $=2, \sigma_{\min }=$ $10^{-10}, \sigma_{\max }=10^{10}, \sigma_{0}=1, \tau_{\min }=0.1, \tau_{\max }=0.5, \gamma=$ $10^{-4}, M=10$, and $\eta_{k}=\left(\left\|F x_{0}\right\| / 2^{k}\right)$ for all $k$.

(2) "N-DF-SANE" means the N-DF-SANE method developed by Cheng and Li [14] with $\eta_{k}=0.6, \sigma_{\min }=$ $10^{-10}, \sigma_{\max }=10^{10}, \rho_{\min }=0.1, \rho_{\max } \gamma=10_{-4}$, and $\varepsilon_{k}=$ $\left(\left\|F\left(x_{0}\right)\right\| / 2^{k}\right)$ for all $k$.

(3) "DF-MLS" stands for Algorithm 1. We set the parameters as follows: $\rho=0.5, \tau_{k}=0.6, t=1, \lambda_{1}=\lambda_{2}=$ $10^{-4}, \alpha_{\min }=10^{-10}, \alpha_{\max }=10^{10}$, and $\eta_{k}=\left(\left\|F\left(x_{0}\right)\right\| /\right.$

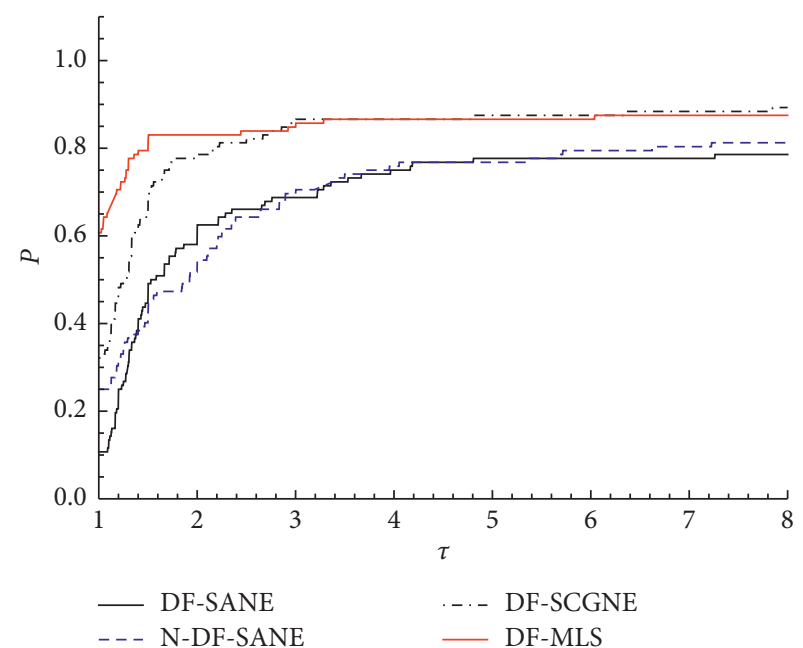

FIGURE 1: Performance profiles related to the number of iterations.

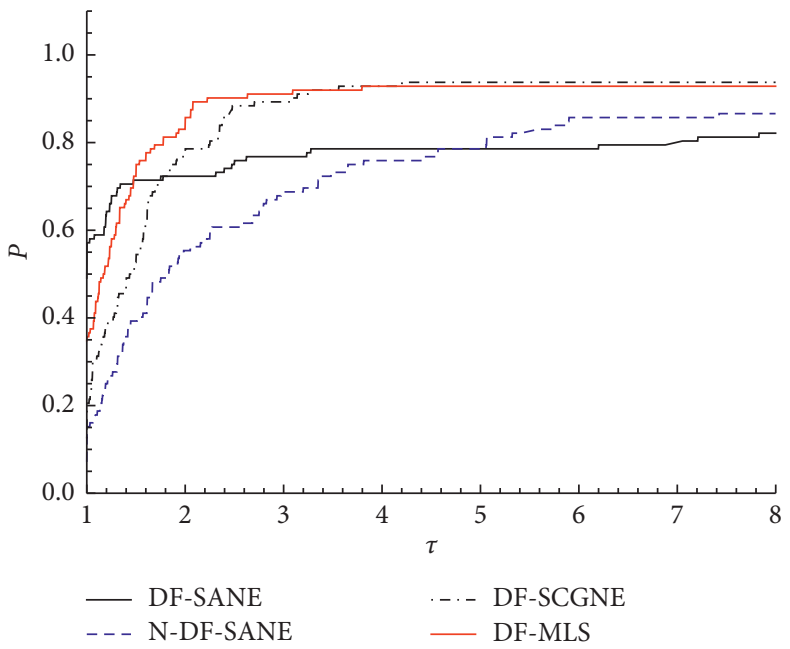

FIgURe 2: Performance profiles related to the number of function evaluations.

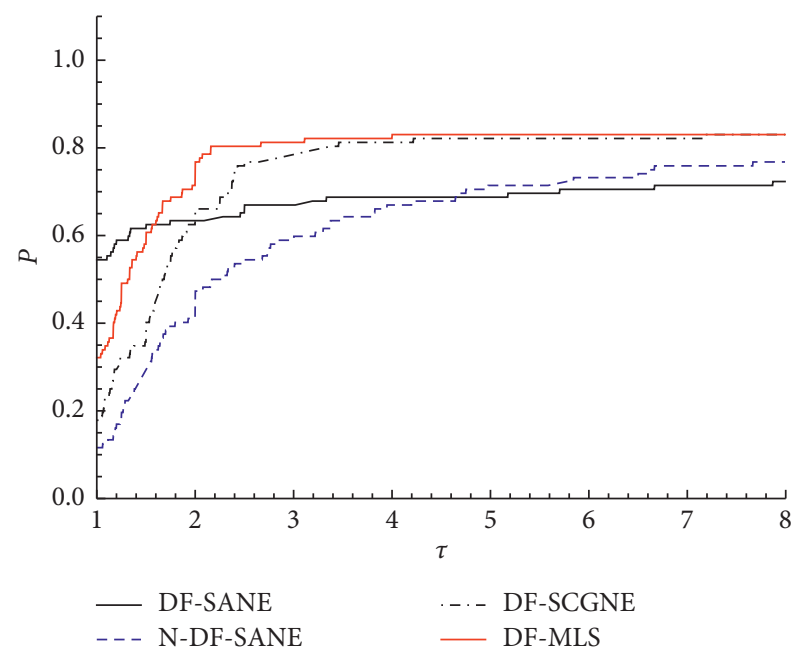

FIgURe 3: Performance profiles relative to the CPU time. 
$\left.2^{k}\right)$ for all $k$. In the line search, the initial step length $\alpha_{k_{0}}$ is determined by

$$
\begin{gathered}
\alpha_{k_{0}}= \begin{cases}\alpha_{\max }, & \text { if } \sigma>\alpha_{\max }, \\
\sigma, & \text { if } \sigma \in\left[\alpha_{\min }, \alpha_{\max }\right], \\
\alpha_{\min }, & \text { if } \sigma<\alpha_{\min },\end{cases} \\
\sigma=\frac{-F_{k}^{T} d_{k}}{d_{k}^{T} z_{k}}, \\
z_{k}=\frac{F\left(x_{k}+\varepsilon d_{k}\right)-F\left(x_{k}\right)}{\varepsilon}, \\
\varepsilon=10^{-8} .
\end{gathered}
$$

(4) "DF-SCGNE" means the DF-SCGNE method proposed by $\mathrm{Yu}$ [34] using the same line search as DFMLS.

For each method, the corresponding curve in Figure 1 plots the fraction $P$ of problems for which the method is within a factor $\tau$ of the smallest total number of iterations. We observe from Figure 1 that DF-MLS solves $60.71 \%$ (68 out of 112) of the test problems with the smallest total number of iterations. This may be because the DF-MLS method can generate more efficiently than other methods. We observe from Figures 1 and 2 that the performance of DF-MLS is better than that of N-DF-SANE and DF-SCGNE. However, the DF-MLS method requires more function evaluations and CPU time than DF-SANE. This might be because that the DF-MLS method needs an additional function evaluation to determine the initial step length $\alpha_{k_{0}}$ at each iteration. Figure 3 compares the performance of these four methods relative to the number of function evaluations. How to improve the efficiency of the DF-MLS method will be an interesting topic for us in the future.

\section{Conclusion}

Many practical applications give rise to nonlinear systems of equations. In the past few decades, Newton's methods [1-10] have been widely used to solve this problem for their fast convergence speeds. However, Newton's methods are not suitable for solving a large-scale problem in which the Jacobian matrix of the objective function is unavailable or needs massive storage space. To overcome this shortcoming of Newton's methods, some derivative-free numerical algorithms have been developed such as the SANE, DF-SANE, and some CG-type methods [11, 13, 29-34].

Inspired by the CG-type methods, we design a derivative-free method for a large-scale nonlinear system of equations based on the MLS [24] conjugate gradient method. The line search in the proposed method is a modification of the Zhang-Hager [15] and Cheng-Li [14] nonmonotone line searches. We established the global convergence based on some mild conditions. The proposed method is suitable to solve large-scale problems because it does not need the Jacobian matrix of the objective function.
In the numerical experiment, we tested 112 unconstrained optimization problems in the CUTEr library [38] and compared the numerical performance of our method with that of DF-SANE [13], N-DF-SANE [14], and DF-SCGNE [34]. We used the methods to find the stationary points of the test problems by using their gradients only. The numerical results show that the proposed method is efficient and has good numerical stability.

\section{Data Availability}

The data used to support the findings of this study are available from the corresponding author upon request.

\section{Conflicts of Interest}

The authors declare that there are no conflicts of interest regarding the publication of this paper.

\section{Acknowledgments}

The authors are very grateful to Prof. Raydan for providing them the codes.

\section{References}

[1] E. G. Birgin, N. Krejic, and J. Martínez, "Globally convergent inexact quasi-Newton methods for solving nonliear systems," Numerical Algorithms, vol. 32, pp. 249-260, 2003.

[2] S. Bellavia and B. Morini, "A globally convergent NewtonGMRES subspace method for systems of nonlinear equations," SIAM Journal on Scientific Computing, vol. 23, no. 3, pp. 940-960, 2001.

[3] P. N. Brown and Y. Saad, "Convergence theory of nonlinear Newton-Krylov algorithms," SIAM Journal on Optimization, vol. 4, no. 2, pp. 297-330, 1994.

[4] M. G. Gasparo, "A nonmonotone hybrid method for nonlinear systems," Optimization Methods and Software, vol. 13, no. 2, pp. 79-94, 2000.

[5] D. Li and M. Fukushima, "A derivative-free line search and global convergence of Broyden-like method for nonlinear equations," Optimization Methods and Software, vol. 13, pp. 583-599, 2000.

[6] J. M. Martínez, "A family of quasi-Newton methods for nonlinear equations with direct secant updates of matrix factorizations," SIAM Journal on Numerical Analysis, vol. 27, no. 4, pp. 1034-1049, 1990.

[7] D. Li and M. Fukushima, "A globally and superlinearly convergent gauss-Newton-based BFGS method for symmetric nonlinear equations," SIAM Journal on Numerical Analysis, vol. 37, no. 1, pp. 152-172, 1999.

[8] M. Solodov and B. Svaiter, "A globally convergent inexact Newton method for systems of monotone equations," in Reformulation: Nonsmooth, Piecewise Smooth, Semismooth and Smoothing Methods, M. Fukushima and L. Qi, Eds., Kluwer Academic, Dordrecht, Netherlands, pp. 355-369, 1998.

[9] G. Zhou and K. C. Toh, "Superlinear convergence of a Newton-type algorithm for monotone equations," Journal of Optimization Theory and Applications, vol. 125, no. 1, pp. 205-221, 2005. 
[10] W. Zhou and D. Li, "Limited memory BFGS method for nonlinear monotone equations," Journal of Computational Mathematics, vol. 25, pp. 89-96, 2007.

[11] W. La Cruz and M. Raydan, "Nonmonotone spectral methods for large-scale nonlinear systems," Optimization Methods and Software, vol. 18, no. 5, pp. 583-599, 2003.

[12] L. Grippo, F. Lampariello, and S. Lucidi, "A nonmonotone line search technique for Newton's method," SIAM Journal on Numerical Analysis, vol. 23, no. 4, pp. 707-716, 1986.

[13] W. La Cruz, J. Martínez, and M. Raydan, "Spectral residual method without gradient information for solving large-scale nonlinear systems of equations," Mathematics of Computation, vol. 75, no. 255, pp. 1429-1449, 2006.

[14] W. Cheng and D.-H. Li, "A derivative-free nonmonotone line search and its application to the spectral residual method," IMA Journal of Numerical Analysis, vol. 29, no. 3, pp. 814-825, 2009.

[15] H. Zhang and W. W. Hager, "A nonmonotone line search technique and its application to unconstrained optimization," SIAM Journal on Optimization, vol. 14, no. 4, pp. 1043-1056, 2004.

[16] M. R. Hestenes and E. Stiefel, "Methods of conjugate gradients for solving linear systems," Journal of Research of the National Bureau of Standards, vol. 49, no. 6, pp. 409-436, 1952.

[17] E. Polak and G. Ribiere, "Note sur la convergence de méthodes de directions conjuguées," Revue française d'informatique et de recherche opérationnelle. Série rouge, vol. 3, no. 16, pp. 35-43, 1969.

[18] B. T. Polyak, "The conjugate gradient method in extremal problems," USSR Computational Mathematics and Mathematical Physics, vol. 9, no. 4, pp. 94-112, 1969.

[19] Y. Liu and C. Storey, "Efficient generalized conjugate gradient algorithms, Part 1: Theory," Journal of Optimization Theory and Applications, vol. 69, pp. 177-182, 1991.

[20] J. C. Gilbert and J. Nocedal, "Global convergence properties of conjugate gradient methods for optimization," SIAM Journal on Optimization, vol. 2, no. 1, pp. 21-42, 1992.

[21] L. Zhang, W. Zhou, and D.-H. Li, "A descent modified PolakRibière-Polyak conjugate gradient method and its global convergence," IMA Journal of Numerical Analysis, vol. 26, no. 4, pp. 629-640, 2006.

[22] G. Yu, L. Guan, and W. Chen, "Spectral conjugate gradient methods with sufficient descent property for large-scale unconstrained optimization," Optimization Methods and Software, vol. 23, no. 2, pp. 275-293, 2008.

[23] L. Zhang, "A new Liu-Storey type nonlinear conjugate gradient method for unconstrained optimization problems," Journal of Computational and Applied Mathematics, vol. 225, no. 1, pp. 146-157, 2009.

[24] M. Li and H. Feng, "A sufficient descent LS conjugate gradient method for unconstrained optimization problems," Applied Mathematics and Computation, vol. 218, no. 5, pp. 1577-1586, 2011.

[25] W. W. Hager and H. Zhang, "A new conjugate gradient method with guaranteed descent and an efficient line search," SIAM Journal on Optimization, vol. 16, no. 1, pp. 170-192, 2005.

[26] S. Babaie-Kafaki, "On optimality of two adaptive choices for the parameter of Dai-Liao method," Optimization Letters, vol. 10, no. 8, pp. 1789-1797, 2016.

[27] S. Babaie-Kafaki and R. Ghanbari, "Two optimal Dai-Liao conjugate gradient methods," Optimization, vol. 64, no. 11, pp. 2277-2287, 2015.
[28] Z. Dai, H. Zhu, and J. Kang, "New technical indicators and stock returns predictability," International Review of Economics \& Finance, vol. 71, pp. 127-142, 2021.

[29] G. Yuan and M. Zhang, "A three-terms Polak-Ribière-Polyak conjugate gradient algorithm for large-scale nonlinear equations," Journal of Computational and Applied Mathematics, vol. 286, pp. 186-195, 2015.

[30] G. Yuan, Z. Meng, and Y. Li, "A modified Hestenes and Stiefel conjugate gradient algorithm for large-scale nonsmooth minimizations and nonlinear equations," Journal of Optimization Theory and Applications, vol. 168, no. 1, pp. 129-152, 2016.

[31] A. A. Bala and P. Kumam, "A descent Dai-Liao conjugate gradient method for nonlinear equations," Numerical Algorithms, vol. 81, no. 1, pp. 197-210, 2019.

[32] A. B. Abubakar and P. Kumam, "An improved three-term derivative-free method for solving nonlinear equations," Computational and Applied Mathematics, vol. 37, no. 5, pp. 6760-6773, 2018.

[33] M. Li, "A derivative-free PRP method for solving large-scale nonlinear systems of equations and its global convergence," Optimization Methods and Software, vol. 29, no. 3, pp. 503514, 2014.

[34] G. Yu, "Nonmonotone spectral gradient-type methods for large-scale unconstrained optimization and nonlinear systems of equations," Pacific Journal of Optimization, vol. 7, no. 2, pp. 387-404, 2011.

[35] Z. Dai and H. Zhu, "A modified Hestenes-Stiefel-type derivative-free method for large-scale nonlinear monotone equations," Mathematics, vol. 8, no. 2, p. 168, 2020.

[36] Q.-R. Yan, X.-Z. Peng, and D.-H. Li, "A globally convergent derivative-free method for solving large-scale nonlinear monotone equations," Journal of Computational and Applied Mathematics, vol. 234, no. 3, pp. 649-657, 2010.

[37] J. M. Ortega and W. C. Rheinboldt, Iterative Solution of Nonlinear Equations in Several Variables, Academic Press, New York, NY, USA, 1970.

[38] I. Bongartz, A. R. Conn, N. Gould, and P. L. Toint, "Cute," ACM Transactions on Mathematical Software, vol. 21, no. 1, pp. 123-160, 1995.

[39] E. D. Dolan and J. J. Moré, "Benchmarking optimization software with performance profiles," Mathematical Programming, vol. 91, no. 2, pp. 201-213, 2002. 